\title{
Sleep Quality in Adolescents: What's Discriminates Good from Poor Sleepers?
}

Sílvia HM Pucci ${ }^{\star}$ and M Graça Pereira

School of Psychology, University of Minho, Braga, Portugal

*Corresponding author: Sílvia HM Pucci, School of Psychology, University of Minho, Braga, Portugal, Tel: +351 253 604228; E-mail: shmpucci@gmail.com

Received date: January 21, 2016; Accepted date: February 26, 2016; Published date: March 04, 2016

Copyright: @ 2016 Pucci SHM, et al. This is an open-access article distributed under the terms of the Creative Commons Attribution License, which permits unrestricted use, distribution, and reproduction in any medium, provided the original author and source are credited.

\section{Abstract}

Background: The study focused on discriminating adolescents with good versus poor sleep quality.

Method: The sample consisted of 272 adolescents and a transversal design was used. The instruments assessed socio-demographic data, psychological morbidity, sleep quality, family sleep behavior, excessive daytime sleepiness, sleep habits and social skills.

Results: The results revealed that age, caffeine intake, psychological morbidity, excessive daytime sleepiness, and family sleep behavior were variables that discriminated good from poorer sleepers in terms of adolescents' sleep quality.

Conclusions: The results emphasize the role of family on sleep quality and the need to include family members in intervention programs. Programs should be sensitive to adolescents' age and gender.

Keywords: Adolescent; Sleep; Psychological morbidity; Caffeine

\section{Introduction}

The phase of adolescence is marked by changes that occur on many different aspects of the individual's life, including changes in sleep patterns [1]. Sleep plays an active role in the brain during an individual's overall development, especially regarding restorative functions [2]. Several studies have reported a trend of insufficient and poor sleep, in adolescents [3,4]. A study that analyzed 5226 adolescents classified $20 \%$ as poor sleepers.

The study showed that the prevalence of poor sleep, increased with age, grade level, and older adolescents showed worse sleep efficiency, problems in falling asleep, and more sleep disturbances [5]. At the same time, a research that focused on disturbed sleep and associated factors, in 1221 adolescents, found that almost 35\% of adolescents showed poor sleep that increased with age [4].

Psychological factors may affect sleep patterns in adolescents, interacting with biological regulatory processes, like autonomy to decide the right time to go to bed, use of technology/electronic equipment, social activities, and parental-set-bedtime [6]. It is possible to extend adolescents' sleep with parent's intervention on set-bedtime; and with the combination of "extended sleep" and "early bedtime"; positive outcomes may be achieved regarding depression, fatigue and sleepiness [6].

There is a range of possible causes and consequences of sleep quality in adolescents, and psychological morbidity seems to be one important and relevant factor. Some studies have linked psychological morbidity, i.e., depression/anxiety, with adolescents' sleep.

In fact, a study that estimated the relationship between anxiety/ depression and sleep, in a non-clinical child sample aged 6 to 11 years old found, through mothers' reports, that children with troubled sleep had higher odds of developing depression and anxiety [7]. Other interesting research [8] found an association between childhood sleep problems and anxiety and depression, later on, in adulthood. The authors found that adulthood's anxiety was predicted by the persistence of sleep problems in adolescence.

Another psychological variable with great relevance to sleep quality in adolescents is family functioning, although there is a lack of studies that focused on the association between these two variables [9]. A study that focused on the perceived sleep quality, health habits, and psychological factors revealed that having a good environment at home was essential for sleep quality and quantity, among adolescents [10].

As indicators of poor sleep quality, are the problems of initiating and maintaining sleep among children and adolescents that are common with varying prevalence's estimations, probably due to the methodology and design of the studies [11]. Excessive daytime sleepiness (EDS) is one of the most common clinical consequences of inadequate sleep, having a big prevalence among teenagers [12].

In fact, daytime sleepiness is closely associated with sleep habits that youngsters develop and follow during adolescence [13] and is related to mood disorders and psychological morbidity [14-16].

Several studies have shown that sleep habits and sleep quality, in adolescents, are related. In fact, sleep habits change according to age, and adjustments in sleep schedules and time spent in bed, need to be done [17]. The use of electronic equipment is an important factor that influences adolescents' sleep. Studies have shown that among the most important impairments for good sleep quality are adolescents' habits, such as watching television [18]. Adolescents with less hours of sleep, who watched television at night, also have a higher consumption of caffeine intake [19].

In fact, psychoactive substances are also related to sleep quality, in adolescents. Caffeine is one of the most consumed substance worldwide, by adults and adolescents. Caffeine is quickly absorbed, 
reaching a plasmatic high in about 30 to 75 minutes when consumed orally [20]. A literature review focusing on caffeine and sleep concluded that in children and adolescents, poor sleep quality and caffeine intake is related [21].

Taking in consideration the literature reviewed, the present study focused on the variables that discriminate adolescents with good versus poor sleep quality [22]. According to the literature, we expect that psychological morbidity, daytime sleepiness and more problems with family sleep behaviors will discriminate poor from good sleepers [23]. Knowing which variables are the most important and discriminating adolescents' sleep quality will help health care professionals to plan interventions, in this population [24].

\section{Method and Material}

\section{Participants}

Participants were 272 adolescents from two public schools, aged between 12 and 18 years old $(\mathrm{M}=15.48, \mathrm{SD}=1.61)$. The majority of students were female (58\%), with married parents (79\%), with mothers (51\%) and fathers (73\%) consuming alcohol. Of the total sample, $31 \%$ had a daily intake of caffeine, $47 \%$ consumed beverages with caffeine and $23 \%$ consumed energetic drinks.

\section{Design and settings}

The study used a transversal design. The research was approved by the General Education Committee. The data were collected in two secondary public schools in the Northern region of Portugal. The participation was voluntary, and the parents signed an informed consent regarding their adolescent's participation. The instruments were completed by adolescents, at school, during class.

The inclusion criteria were 1) age between 12 and 18;2) being a student in the class the day of the assessment; 3 ) parent's approval to be part of the study. Exclusion criteria included parent's non-approval of the adolescent's inclusion in the study.

\section{Assessments}

Pittsburgh sleep quality index (PSQI): This instrument assesses sleep quality during the previous month and has 19 items [25]. PSQI is composed of seven components that evaluate daytime dysfunction, sleep medication, sleep disturbances, sleep efficiency, sleep duration, sleep latency and sleep quality. In the present study only the total scale was used and the Cronbach's alpha was 0.60.

Modified excessive sleepiness scale (MESSA): The instrument assesses the propensity of adolescents to fall asleep in different situations and is composed of 13 items [26]. In this study, only the total scale was used and the Cronbach's alpha for the total scale was .78.

Hospital anxiety and depression scales (HADS): The instrument assesses the levels of depression and anxiety [27]. It has 14 items that give a psychological morbidity global score. There are two subscales (anxiety and depression). The cutoff score for clinical depression and anxiety is 8. In this study, only the total scale was used and the Cronbach's alpha was 0.76 .

Howard family sleep questionnaire (HFSQ): This scale assesses the cumulative impact of family dynamics on sleep behavior and is composed of 52 items that assess physical and cultural aspects of the family environment that influence sleep (e.g. "we eat dinner together", "we respect each other's needs for quiet once one of us goes to bed", "on weekends, we have no set bedtime [28]. In this study, Cronbach's alpha was 0.70 .

Sleep habits questionnaire (SHQ): This 12 item questionnaire assesses the impact of behaviors that may influence sleep in adolescents (e.g. how many times a week do you play video-games after bedtime, how many times a week do you read, study or, do homework after bedtime) [29]. In the present study, Cronbach's alpha was 0.70 .

\section{Data extraction/scoring}

Data was abstracted from the instruments that were scored regarding the original instructions of their authors i.e., summing the items according to the Likert scale. In the Pittsburgh Sleep Quality Index, Howard Family Sleep Questionnaire, and the Sleep Habits Questionnaire, a higher score indicates poor sleep quality, poor family sleep behaviors, and poor sleep habits, respectively.

Regarding the Modified Excessive Sleepiness Scale, Hospital Anxiety and Depression Scales, a higher score indicates respectively the presence of excessive daytime sleepiness, and more anxiety and depression. Information regarding age, gender, the consumption of caffeine and energetic beverages was abstracted from a sociodemographic questionnaire.

\section{Data analysis}

A discriminant analysis was used to determine which variables differentiated adolescents with good versus poor sleep quality based on the sample median (stepwise method).

This statistical procedure determines which variables differentiate the two groups allowing to know the best predictors of sleep quality. The stepwise procedure in particular allows seeing which variables are included in the prediction of group membership (good versus poorer sleepers).

\section{Results}

The results showed that age, caffeine intake, psychological morbidity, daytime sleepiness and family sleep behaviors discriminated adolescents who had good versus poor sleep quality (Table 1). Low function values indicate good sleep quality (mean $=-0.354)$ and higher function values indicate poor sleep quality (mean $=0.519$ ). Therefore, lower values of the discriminant function belong to the good sleep quality group.

The variables that present the highest coefficient in absolute value are the most important. Therefore, adolescents with psychological morbidity (-0.619) and family sleep behavior (-0.455), i.e., less depression/anxiety and less problems with family sleep behaviors belong to the good sleepers group. Adolescents with more excessive daytime sleepiness (0.435), being older (0.370) and drinking more caffeine beverages $(0.335 ; 0.328)$ belong to the poorer sleepers.

Based on the results, groups were significantly different and the discriminant function was significant (Wilk's Lambda $=0.844$; Qi2 $=$ 44.83; $\mathrm{p}<0.000$ ) (Table 2). The classification matrix identified $66.5 \%$ of cases as correctly classified, i.e., in group $0,79.6 \%$ are good sleepers and in group 1, $45.5 \%$ are poor sleepers. 
Page 3 of 5

\begin{tabular}{|c|c|c|c|c|c|c|c|}
\hline \multirow{2}{*}{ Variables } & \multicolumn{2}{|c|}{ Good sleepers } & \multicolumn{2}{|c|}{ Poor sleepers } & \multirow[t]{2}{*}{ Wilks' Lambda } & \multirow[t]{2}{*}{$\mathbf{F}$} & \multirow[t]{2}{*}{$\mathbf{P}$} \\
\hline & Mean & SD & Mean & SD & & & \\
\hline Age & 15.26 & 1.7 & 15.77 & 1.3 & 0.975 & 6.776 & 0.01 \\
\hline Caffeine Intake & 1.25 & 0.46 & 1.40 & 0.57 & 0.98 & 5.319 & 0.022 \\
\hline Use of Energy Drinks & 1.22 & 0.59 & 1.41 & 0.7 & 0.98 & 5.537 & 0.019 \\
\hline HADS (Depression/Anxiety) & 20.06 & 3.29 & 18.23 & 3.5 & 0.934 & 18.92 & 0 \\
\hline MESSA (Daytime Sleepiness) & 11.63 & 6.1 & 13.95 & 6.0 & 0.966 & 9.336 & 0.002 \\
\hline HFSQ (Family Sleep) & 99.95 & 10.9 & 95.75 & 10.1 & 0.963 & 10.222 & 0.002 \\
\hline
\end{tabular}

Table 1: Significant psychosocial variables that discriminate poor from good sleep quality in adolescents.

\begin{tabular}{|l|l|}
\hline Sleep Quality & Function \\
\hline HADS & -0.619 \\
\hline HFSQ & -0.455 \\
\hline MESSA & 0.435 \\
\hline Age & 0.37 \\
\hline Energy Drinks & 0.335 \\
\hline Caffeine Intake & 0.328 \\
\hline
\end{tabular}

there were no differences on caffeine and energy drink consumption between the two groups [31].

On the other hand, a more recent study with 225 adolescents concluded that $79 \%$ drank caffeine and that higher intake of caffeine was negatively associated with sleep quality [32], which was corroborated by other studies [19].

Family variables were an important discriminant factor between good and poor sleep quality. In line with our results, a recent study analyzed the relationship between parents and adolescent sleep patterns and psychological functioning, finding a relationship between parents and children sleep patterns; and a relationship between stress perception, depression symptoms, family environment and subjective sleep disturbances in adolescents. Adolescents' sleep disturbances were independently predicted by stress, perceived negative parental styles and mother's poorer sleep [33]. Another study found a significant association for parents and adolescents regarding sleep patterns sleep disturbances, depression and family environment. In the face of these results, the authors concluded that there might be a bidirectional association between family function, family environment, well-being and adolescent's sleep, and that mothers and children sleep and wellbeing are related [34]. Our results are in accordance with this literature, emphasizing the important role of parents in promoting sleep quality with adolescents.

This study found that psychological morbidity was a very important variable discriminating adolescents' sleep quality. In fact, several studies have shown the association between sleep problems and psychological symptoms, in adolescents $[2,35]$. A study analyzed the relationship between sleep and suicidal behaviors in students and concluded that sleep disturbances were associated with suicidal ideation [36]. Interestingly, after adjusting for the statistical effect of depression, this relationship ceased to exist, leading the authors to hypothesize that it was mediated by depression.

Another study that assessed sleep quality as a predictor of sleep patterns found that lower sleep quality was related to depressions selfreports [31]. An association between sleep problems and anxiety and depression was also found in another study, showing that emotional problems could be predicted by sleep problems [37]. This relationship between sleep quality and psychological morbidity seems to be bidirectional. In any case, psychological morbidity either a cause or a consequence of poor sleep quality, is an important variable to be addressed in future interventions. 
This study emphasizes how important variables such as caffeine intake and family sleep behaviors, that are less researched, need to be addressed and incorporated in health promotion interventions regarding sleep quality, in adolescents.

\section{Limitation}

The Cronbach alpha of the Pittsburgh Sleep Questionnaire Index was acceptable but low 0.60 (total scale). Finally, the use of only selfreported instruments and the cross-sectional design are also limitations.

\section{Conclusion/Clinical Implications}

This study concluded that age, caffeine intake, psychological morbidity, daytime sleepiness and the influence of family on sleep behaviors were discriminant factors for sleep quality among adolescents. It is crucial to take these variables into consideration in health promotion programs with this population.

Psychological morbidity and family factors were important discriminating variables in adolescent sleep quality. Intervention with teenagers should, therefore, pay attention to depression and anxiety symptoms and should include the family, in order to promote better sleep quality.

Systemic intervention is crucial to promote healthy habits in the family, addressing in particular, the consumption of caffeine and other psychoactive substances, and family sleep behaviors that impacts adolescents sleep quality.

Future studies should focus on detecting poor sleep quality causes, in order to understand possible bidirectional relationships and comorbidities and use longitudinal designs to understand the influence of these variables, over time.

\section{Acknowledgement}

The present research had a support of Erasmus Mundus 15.

\section{Disclosure}

No author indicated any conflicts of interest.

\section{References}

1. Carskadon MA, Vieira C, Acebo C (1993) Association between puberty and delayed phase preference. Sleep 16: 258-262.

2. Dahl RE, Lewin DS (2002) Pathways to adolescent health sleep regulation and behavior. J Adolesc Health 31: 175-184.

3. Wolfson AR, Carskadon MA (1998) Sleep schedules and daytime functioning in adolescents. Child Dev 69: 875-887.

4. Zhou HQ, Shi WB, Wang XF, Yao M, Cheng GY, et al. (2012) An epidemiological study of sleep quality in adolescents in South China: a school-based study. Child Care Health Dev 38: 581-587.

5. Xu Z, Su H, Zou Y, Chen J, Wu J, et al. (2012) Sleep quality of Chinese adolescents: distribution and its associated factors. J Paediatr Child Health 48: 138-145.

6. Carskadon MA (2011) Sleep in adolescents: the perfect storm. Pediatr Clin North Am 58: 637-647.

7. Johnson EO, Chilcoat HD, Breslau N (2000) Trouble sleeping and anxiety/depression in childhood. Psychiatry Res 94: 93-102.

8. Gregory AM, Caspi A, Eley TC, Moffitt TE, O'Connor TG, et al. (2005) Prospective Longitudinal Associations Between Persistent Sleep Problems in Childhood and Anxiety and Depression Disorders in Adulthood. J Abnorm Child Psych 33: 157-163.

9. Adam EK, Snell EK, Pendry P (2007) Sleep timing and quantity in ecological and family context: a nationally representative time-diary study. J Fam Psychol 21: 4-19.

10. Tynjälä J, Kannas L, Levälahti E, Välimaa R (1999) Perceived sleep quality and its precursors in adolescents. Health Promot Int 14: 155-166.

11. Dewald JF, Meijer AM, Oort FJ, Kerkhof GA, Bögels SM (2010) The influence of sleep quality, sleep duration and sleepiness on school performance in children and adolescents: A meta-analytic review. Sleep Med Rev 14: 179-189.

12. Millman RP; Working Group on Sleepiness in Adolescents/Young Adults; AAP Committee on Adolescence (2005) Excessive sleepiness in adolescents and young adults: causes, consequences, and treatment strategies. Pediatrics 115: 1774-1786.

13. Cain N, Gradisar M (2010) Electronic media use and sleep in school-aged children and adolescents: A review. Sleep Med 11: 735-742.

14. Carskadon MA (1990) Patterns of sleep and sleepiness in adolescents. Pediatrician 17: 5-12.

15. Carskadon MA, Wolfson AR, Acebo C, Tzischinsky O, Seifer R (1998) Adolescent sleep patterns, circadian timing, and sleepiness at a transition to early school days. Sleep $21: 871-881$.

16. Leotta C, Carskadon M, Acebo C, Seifer R, Quinn B (1997) Effects of acute sleep restriction on affective response in adolescents: Preliminary results. Sleep Res 26: 201.

17. Colrain IM, Baker FC (2011) Changes in sleep as a function of adolescent development. Neuropsychol Rev 21: 5-21.

18. Noland H, Price JH, Dake J, Telljohann SK (2009) Adolescents' sleep behaviors and perceptions of sleep. J Sch Health 79: 224-230.

19. Lodato F, Araújo J, Barros H, Lopes C, Agodi A, et al. (2013) Caffeine intake reduces sleep duration in adolescents. Nutr Res 33: 726-732.

20. Mandel HG (2002) Update on caffeine consumption, disposition and action. Food Chem Toxicol 40: 1231-1234.

21. Roehrs T, Roth T (2008) Caffeine: sleep and daytime sleepiness. Sleep Med Rev 12: 153-162.

22. Buysse DJ, Reynolds CF 3rd, Monk TH, Berman SR, Kupfer DJ (1989) The Pittsburgh Sleep Quality Index: a new instrument for psychiatric practice and research. Psychiatry Res 28: 193-213.

23. Billings T, Berg-Cross L (2010a) The Modified Epworth Sleepiness Scale for Adolescents: A Brief Measure of Daytime Sleepiness in Adolescents. Howard University, Washington, DC.

24. Zigmond AS, Snaith RP (1983) The hospital anxiety and depression scale. Acta Psychiatr Scand 67: 361-370.

25. Billings T, Bangash O, Berg-Cross L (2010) Predicting Sleep Problems in Adolescents: Validation of the Family Sleep Questionnaire. Howard University, Washington.

26. Billings T, Berg-Cross L (2010b) Sleep Competing Activities and Sleep Problems. Howard University, Washington, DC.

27. Gradisar M, Gardner G, Dohnt H (2011) Recent worldwide sleep patterns and problems during adolescence: a review and meta-analysis of age, region, and sleep. Sleep Med 12: 110-118.

28. Yang CK, Kim JK, Patel SR, Lee JH (2005) Age-related changes in sleep/ wake patterns among Korean teenagers. Pediatrics 115: 250-256.

29. Taylor DJ, Jenni OG, Acebo C, Carskadon MA (2005) Sleep tendency during extended wakefulness: insights into adolescent sleep regulation and behavior. J Sleep Res 14: 239-244.

30. Urschitz MS, Heine K, Brockmann PE, Peters T, Durst W, et al. (2013) Subjective and objective daytime sleepiness in schoolchildren and adolescents: results of a community-based study. Sleep Med 14: 1005-1012.

31. Lund HG, Reider BD, Whiting AB, Prichard JR (2010) Sleep patterns and predictors of disturbed sleep in a large population of college students. J Adolesc Health 46: 124-132.

32. Anderson BL, Juliano LM (2012) Behavior, sleep, and problematic caffeine consumption in a college-aged sample. J Caffeine Res 2: 38-44. 
Citation: Pucci SHM, Pereira MG (2016) Sleep Quality in Adolescents: What's Discriminates Good from Poor Sleepers?. J Sleep Disord Ther 5: 237. doi:10.4172/2167-0277.1000237

Page 5 of 5

33. Bajoghli H, Alipouri A, Holsboer-Trachsler E, Brand S (2013) Sleep patterns and psychological functioning in families in northeastern Iran evidence for similarities between adolescent children and their parents. J Adolescence 36: 1103-1113.

34. Kalak N, Gerber M, Kirov R, Mikoteit T, Pühse U, et al. (2012) The relation of objective sleep patterns, depressive symptoms, and sleep disturbances in adolescent children and their parents: A sleep-EEG study with 47 families. J Psychiat Res 46: 1374-1382.
35. Johnson EO, Roth T, Breslau N (2006) The association of insomnia with anxiety disorders and depression: exploration of the direction of risk. J Psychiatr Res 40: 700-708.

36. Liu X (2004) Sleep and adolescent suicidal behavior. Sleep 27: 1351-1358.

37. Gregory AM, O'Connor TG (2002) Sleep problems in childhood: a longitudinal study of developmental change and association with behavioral problems. J Am Acad Child Adolesc Psychiatry 41: 964-971. 